\title{
Prediction of Lightning Inception by Large Ice Particles and Extensive Air Showers
}

\author{
Anna Dubinova, ${ }^{1, *}$ Casper Rutjes, ${ }^{1, \dagger}$ Ute Ebert, ${ }^{1,2}$ Stijn Buitink, ${ }^{3}$ Olaf Scholten, ${ }^{3,4}$ and Gia Thi Ngoc Trinh ${ }^{4}$ \\ ${ }^{1}$ Centrum Wiskunde \& Informatica (CWI), Amsterdam, Netherlands \\ ${ }^{2}$ Eindhoven University of Technology, Eindhoven, Netherlands \\ ${ }^{3}$ Vrije Universiteit Brussel, Brussels, Belgium \\ ${ }^{4}$ KVI-CART, University of Groningen, Groningen, Netherlands
}

(Received 9 March 2015; published 30 June 2015)

\begin{abstract}
We derive that lightning can start if the electric field is $15 \%$ of the breakdown field, and if elongated ice particles of $6 \mathrm{~cm}$ length and 100 free electrons per $\mathrm{cm}^{3}$ are present. This is one particular example set from a parameter range that we discuss as well. Our simulations include the permittivity $\epsilon(\omega)$ of ice. 100 free electrons per $\mathrm{cm}^{3}$ exist at $5.5 \mathrm{~km}$ altitude in air showers created by cosmic particles of at least $5 \times 10^{15} \mathrm{eV}$. If the electric field zone is $3 \mathrm{~m}$ high and $0.2 \mathrm{~km}^{2}$ in the horizontal direction, at least one discharge per minute can be triggered. The size distribution of the ice particles is crucial for our argument; more detailed measurements would be desirable.
\end{abstract}

DOI: 10.1103/PhysRevLett.115.015002

PACS numbers: $52.80 . \mathrm{Mg}, 92.60 . \mathrm{Pw}$

Introduction.-Lightning inception is the first out of the "top ten questions in lightning research" according to a recent review [1]. How can lightning start when the electric fields in thunderclouds are well below the classical breakdown field $E_{k}$ [2] that is required for electron multiplication and ionization growth? And when the height of the high electric field zone is typically smaller than a kilometer?

It was already suggested a few decades ago that a discharge could start in a lower electric field due to the relativistic runaway electron breakdown: cosmic particles could create ionization avalanches of relativistic particles when the electric field exceeds the threshold field $E_{\text {RREA }} \approx$ $E_{k} / 10$ for the formation of a relativistic runaway electron avalanche (RREA). However, as the RREA length is of the order of $300 \mathrm{~m}$ for a field of $2.8 \mathrm{kV} / \mathrm{cm}$ (at standard temperature and pressure) [3], the electric field needs to exceed $E_{\text {RREA }}$ over heights of several $\mathrm{km}$.

Another suggestion is that hydrometeors, i.e., airborne particles consisting of liquid or frozen water (droplets, snowflakes, graupel, hail, etc.) could enhance the electric field locally in their neighborhood due to their high permittivity [4]. Experiments show how air discharges start from ice particles [5,6]; however, the background electric fields here are as large as $0.3 E_{k}$, and the free electrons needed to start the discharge are created through a radioactive source. In Ref. [7] an ice particle is modeled as an ionized patch of air. This model demonstrates the field enhancement around a real hydrometeor and the emergence of a discharge, but the electrons are trivially available from the ionized patch while a lack of free electrons is an essential issue in a thundercloud.

Free electrons in the high field region are needed to start a discharge. They are generated up to a few $\mathrm{km}$ in altitude by the decay of radioactive elements emitted from the ground, and furthermore by solar energetic particles and by cosmic rays. However, within the troposphere these free electrons attach within tens of nanoseconds to oxygen molecules and form roughly $10^{3}$ positive and negative ions per $\mathrm{cm}^{3}$. In dry air, the electrons can detach again and start a discharge when the electric field exceeds $E_{k}[8,9]$. But in humid air, the $\mathrm{O}_{2}^{-}$ions attract water molecules within microseconds [10]. The electron detachment time from such ion-water clusters is of the order of micro- or even milliseconds [11], and it is negligible on the nanosecond time scale of the primary discharge evolution. Gurevich and Karashtin [12] suggested that the free electrons near a hydrometeor could be supplied by RREAs in air showers created by cosmic particles with energies between $10^{11}$ and $10^{12} \mathrm{eV}$. However, they do not elaborate whether a discharge would actually start-according to our analysis below it wouldn't - and their frequency of cosmic particles is 2 or 3 orders of magnitude smaller than in the Particle Data Group [13]. Furthermore, the frequency dependence of the dielectric permittivity $\epsilon(\omega)$ of ice has to be taken into account when calculating the field enhancement near a frozen hydrometeor-it is 90 for static electric fields, but only 3 for fields changing on a nanosecond time scale.

Structure of the approach.-Whether lightning can be started by an extensive air shower hitting a hydrometeor, depends (i) on the distributions of hydrometeor sizes and shapes, (ii) on the distribution of electric fields in the thundercloud, and (iii) on the distribution and properties of extensive air showers created by high energy particles penetrating the atmosphere. Here we determine one set of parameters in this high-dimensional space that is likely to start lightning.

We start with analyzing the requirements on hydrometeor sizes and shapes, on background electron density and on background electric field to start a discharge, and we perform simulations showing that and how the discharge 
actually starts under these conditions. Then we analyze the energy of the galactic cosmic particles required to create the necessary density of free electrons. Finally, we investigate the probability that the requirements on hydrometeors, electric fields, and cosmic particles coincide.

The altitude for our calculations is $5.5 \mathrm{~km}$, a typical altitude for lightning inception [14]. According to the International Standard Atmosphere, we assume $T=250 \mathrm{~K}$, $p=500$ mbar, and hence an air density $n=0.6 n_{0}$, where $n_{0}$ is at ground level. The transport and reaction coefficients (electron mobility and diffusion and effective Townsend coefficient including 2- and 3-body attachment) for an air discharge are calculated with BOLSIG+ [15] with Phelps' database [16].

Requirements on hydrometeor size and shape as a function of the background field.-A frozen hydrometeor moving in a thundercloud electric field that changes on a millisecond time scale or more slowly will locally enhance the field due to its high dielectric permittivity $\epsilon=90$. To start a self-propagating streamer discharge, a free electron needs sufficiently many multiples of the ionization length. This ionization length as a function of the local electric field $E$ is given by the inverse of the effective Townsend coefficient $\alpha_{\text {eff }}(E)$, which is basically the balance of electron impact ionization and electron attachment (hence $\alpha_{\text {eff }}\left(E_{k}\right)=0$ defines the breakdown field $E_{k}$ ). The electron avalanche multiplication factor $e^{M}$ along a given path is given by the Meek number $M=\int \alpha_{\mathrm{eff}}(E) d z$ that is widely used in electrical engineering. In our simulations, a Meek number of 10 was sufficient, and we take that number as a benchmark.

Hydrometeors appear in a large variety of shapes, yet their shape in the direction perpendicular to the thundercloud field does not contribute much to the field enhancement at their tip. Essentially the length of a hydrometeor $\ell$ and its radius of curvature $R$ at the tip parallel to the field determine the electric field enhancement near the tip. Therefore, we approximate the hydrometeor as a prolate ellipsoid of revolution with length $\ell$ and radius of curvature $R$. (In this case the predischarge field can be calculated analytically [17].) For a Meek number of $M_{0}=10$, the hydrometeor length $\ell$ required to start a discharge is a function of the reduced background electric field $E_{\mathrm{bg}} / E_{k}$ and the $R / \ell$ ratio, and is given by $\ell=M_{0} n_{0} / n \times F(R / \ell$, $\left.E_{\mathrm{bg}} / E_{k}\right)$. The Meek number is calculated on the symmetry axis where the field is above the breakdown value $E_{k}$.

Figure 1 shows, as a result, the conditions for a discharge to start at $5.5 \mathrm{~km}$ altitude. The contour lines indicate the lines of constant hydrometeor length $\ell$ as a function shape parameter $R / \ell$ and the reduced thundercloud field $E_{\mathrm{bg}} / E_{k}$. The lines are altitude dependent, and only the length $\ell$ has to be rescaled. At $0 \mathrm{~km}$, the lines are from left to right $3.4 \mathrm{~cm}, 1.7 \mathrm{~cm}, 0.85 \mathrm{~cm}$, and $0.43 \mathrm{~cm}$. At $8 \mathrm{~km}$, they are $7 \mathrm{~cm}, 3.6 \mathrm{~cm}, 1.8 \mathrm{~cm}$, and $0.95 \mathrm{~cm}$, respectively. Lightning inception is possible either for large

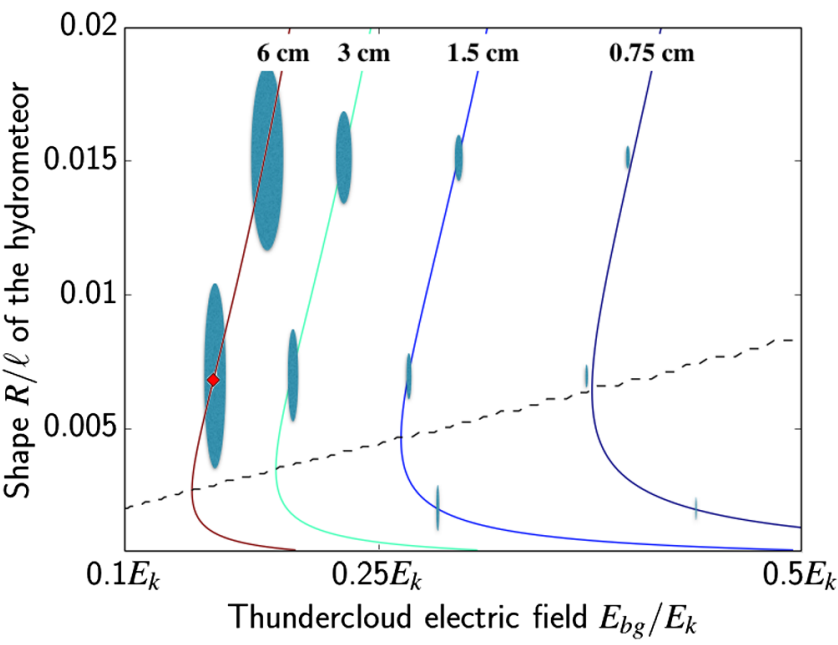

FIG. 1 (color online). Conditions of discharge inception at $5.5 \mathrm{~km}$ altitude. The lines of constant hydrometeor length $\ell$ show when a discharge can start. The axes show the reduced thundercloud field $E_{\mathrm{bg}} / E_{k}$ and the shape parameter $R / \ell$. The red diamond indicates the case in Fig. 2. The dashed curve indicates the optimal $R / \ell$ ratio for given $E_{\mathrm{bg}} / E_{k}$. The ellipsoids are drawn to scale to illustrate the hydrometeor shape and length.

hydrometeors (the left part in Fig. 1) or for high electric fields (the right part). In other words, to create a sufficiently large number of electron multiplications, a hydrometeor in a given thundercloud field not only has to be sufficiently sharp to significantly enhance the field, but also sufficiently long to enhance it in a large region. The optimal aspect ratio $R / \ell$ for given $E_{\mathrm{bg}} / E_{k}$ is indicated by the dashed curve. For a given length $\ell$, hydrometeors sharper than optimal are less likely to initiate a discharge, even though they have a higher electric field directly at the tip.

Below we present the full analysis for one case indicated by the red diamond in Fig. 1. In our simulations, we chose the parameters to minimize the electric field within the parameter space, and therefore the size of the hydrometeor had to be on the extreme side in a thundercloud. Yet, such hydrometeors are observed with a density of roughly $0.1 \mathrm{~m}^{-3}$ [18].

Requirement on the density of free electrons.-The Meek number analysis can be applied if there is at least one free electron ahead of the positive end of the hydrometeor at such a distance that it can drift toward it within its time. For an air density of $n=0.6 n_{0}$, the effective electron lifetime (based on attachment and impact ionization times) is approximately $30 \mathrm{~ns}$. By tracing the electron drift from the hydrometeor surface backward in time for half the lifetime, we found that they came from a volume with $1.5 \mathrm{~mm}$ radius and length, i.e., from a volume of $10 \mathrm{~mm}^{3}$. This means that with a homogeneous density of 100 free electrons per $\mathrm{cm}^{3}$, on average one electron will be available within the relevant volume to start the discharge.

Simulation of actual discharge inception from the hydrometeor.-The discussion above suggests that a positive streamer (developing subsequently into a lightning 
leader) can start from a hydrometeor with a radius of curvature of $R=0.4 \mathrm{~mm}$ and $6 \mathrm{~cm}$ length in a thundercloud field of $2.7 \mathrm{kV} / \mathrm{cm}$ at $5.5 \mathrm{~km}$ altitude when the density of free electrons is initially at least $100 \mathrm{~cm}^{-3}$. We now take these parameters as an input for our 3D cylindrically symmetric discharge model and investigate whether a streamer discharge actually forms and propagates.

The discharge is modeled with the classical diffusiondrift-reaction model of Ref. [19] with space charge effects, and with photoionization included as in Ref. [20].

The hydrometeor is modeled as a dielectric; the dielectric function $\epsilon(\omega)$ of ice depends on frequency $\omega$, it is 90 for slow responses and 3 on the nanosecond time scale [21]. We assume that electrons, when reaching the hydrometeor, attach to the surface. No transport, reactions, or secondary electron emission are assumed on its surface.

The length of the simulation domain is $8.5 \mathrm{~cm}$ and its diameter is $4 \mathrm{~cm}$, sufficiently much larger than the hydrometeor that the background field can be fixed by appropriate Dirichlet boundary conditions for the electric potential on the boundary. Together with the hydrometeor with dielectric constant 90, this fixes the stationary field. The discharge plasma develops its own electric field. Because of the superposition principle, this can be calculated from the charge distribution within the discharge with a constant potential on the outer boundary, and with a dielectric response of the hydrometeor with $\epsilon=3$, and then added to the other field.

The equations are discretized on a static nonuniform grid. The grid is refined in the area where a streamer is expected to propagate. The size of the finest grid cells is $1 \mu \mathrm{m}$. Away from the area of streamer propagation, grid cells quadratically increase in size up to $0.2 \mathrm{~mm}$ on the boundaries.

The result of our simulations is shown in Fig. 2. The left panel shows the electron density and the right panel the electric field strength after 46 ns. Clearly a streamer discharge with its strong field enhancement ahead of the tip has formed below the hydrometeor. The streamer incepts after about $25 \mathrm{~ns}$, leaves the area of enhanced electric field, and propagates due to its self-generated field enhancement into a region where the field is below the breakdown field. The average streamer velocity is about $10^{5} \mathrm{~m} / \mathrm{s}$. The small $\epsilon$ of ice on the ns time scale substantially hinders the streamer propagation; if we would erroneously take $\epsilon=90$ on all time scales, the streamer would propagate twice as fast. As the ice responds with a small $\epsilon$ to the rapid streamer evolution, the field penetrates into the tip of the hydrometeor, as the right panel of Fig. 2 shows.

Occurrence rate of required electron density.-Without assuming a sufficiently high thunderstorm electric field of sufficient extension to form relativistic runaway electron avalanches as in Refs. [12,22], energetic cosmic particles are able to produce enough free electrons in so-called
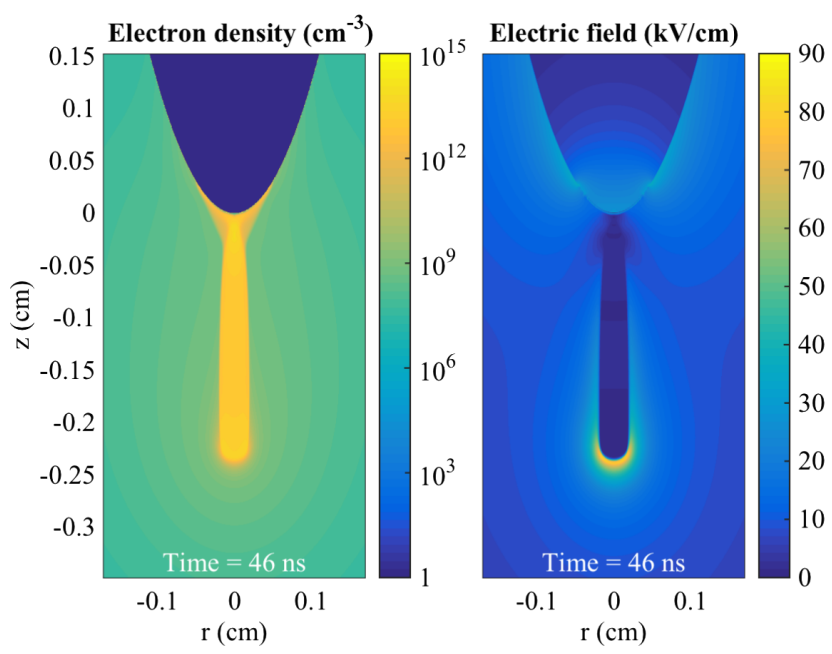

FIG. 2 (color online). Streamer below an icy hydrometeor of length $\ell=6 \mathrm{~cm}$ and curvature radius $R=0.4 \mathrm{~mm}$ in a background field of $E_{\mathrm{bg}}=0.15 E_{k}$ at $5.5 \mathrm{~km}$ altitude. Left: the electron density (green to yellow) with the hydrometeor (blue). Right: The electric field strength. Both figures zoom into $r \leq 2 \mathrm{~mm}$ and $-0.35 \mathrm{~cm} \leq z \leq 0.15 \mathrm{~cm}$ after $46 \mathrm{~ns}$ of simulation.

extensive air showers [13]. We will now calculate the occurrence rate of such events based on models developed for cosmic ray physics.

We focus our analysis on protons with energies between $5 \times 10^{15}$ and $5 \times 10^{16} \mathrm{eV}$. Our calculations described below show that below $5 \times 10^{15} \mathrm{eV}$ it is very unlikely that the core of an extensive air shower reaches a density of 100 thermal electrons per $\mathrm{cm}^{3}$ at $5.5 \mathrm{~km}$ altitude, while above $5 \times 10^{16} \mathrm{eV}$ the electron density is always sufficient, but the occurrence rate decreases with the energy $E$ of the cosmic particle as $E^{-2}$.

Cosmic protons with energies between $5 \times 10^{15}$ and $5 \times 10^{16} \mathrm{eV}$ first interact with an air molecule at about 15 to $25 \mathrm{~km}$ altitude, which marks the beginning of the shower. The number of secondary particles in the shower then increases until it reaches a maximum between 4 and $8 \mathrm{~km}$ altitude, depending on the energy of the primary particle, on the inclination of the particle trajectory with respect to the vertical axis and on the altitude of first interaction. The shower develops downward with nearly the speed of light, and resembles a disc of high energetic particles that leaves a trail of nonrelativistic particles behind. The shower has a narrow core with a very high particle concentration.

Extensive air showers can be simulated by the Monte Carlo program CORSIKA [23] which follows particle movement and interactions explicitly down to a user defined energy threshold of at least $50 \mathrm{keV}$. However, we need the density of free electrons in the $\mathrm{eV}$ range that could start the discharge shown in Fig. 2. So we need to follow the particle generation and motion below CORSIKA's energy threshold. As the cross sections for impact ionization by electrons and positrons are 4 orders of magnitude larger than for 
photoionization, while the photon number is only a factor 20 larger, we only calculate the lower energy electrons generated by electrons and positrons. Furthermore, we use the fact that electrons and positrons of $1 \mathrm{MeV}$ or less can not travel for more than 1 meter at $5.5 \mathrm{~km}$ altitude [24], and we only post-process the electrons and positrons within 3 meters from the core center to derive the electron density within the core. The particle density above this threshold is calculated using the restricted collisional stopping power [25] divided by the net cost per ionization of about 20-30 eV (that depends on energy as elaborated in [24]), this strategy is in line with Ref. [22]. This combination of approaches determines the thermalized free electron density within the core of each shower.

We have simulated 297 showers with primary proton energies between $5 \times 10^{15}$ and $5 \times 10^{16} \mathrm{eV}$ and with random inclinations, and we have determined the flux of electrons and positrons with energy above $1 \mathrm{MeV}$ in the shower core at $5.5 \mathrm{~km}$ altitude. A typical output is 10 to 40 million particles with roughly $90 \%$ photons, $4 \%$ electrons, and $4 \%$ positrons. From these we determined the density of electrons with $\mathrm{eV}$ energy within the core of 1 meter radius according to the prescription above. Taking the measured differential cosmic ray flux [13] into account, the density of 100 thermal electrons per $\mathrm{cm}^{3}$ in the core is reached with a frequency of at least $5 \mathrm{~km}^{-2} \mathrm{~min}^{-1}$ within our sample.

Probability of coincidence of large hydrometeor and extensive air shower inside the thundercloud field.-As sketched in Fig. 3, we require three phenomena to

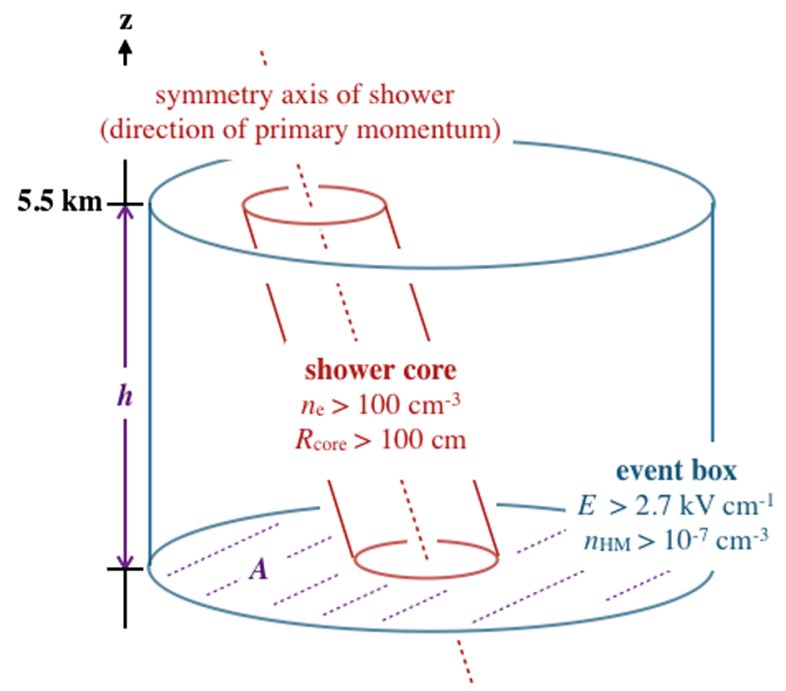

FIG. 3 (color online). Sketch of the model ingredients; not to scale. In the event box (blue) at an altitude of $5.5 \mathrm{~km}$ we assume an electric field $E \geq 2.7 \mathrm{kV} \mathrm{cm}^{-1}$, a density $n_{\mathrm{HM}} \geq 10^{-7} \mathrm{~cm}^{-3}$ of hydrometeors of correct shape and size, and at least one extensive air shower creating a thermalized free electron density $n_{e} \geq$ $100 \mathrm{~cm}^{-3}$ in a core (red) of radius $\geq 100 \mathrm{~cm}$. The height $h$ and horizontal area $A$ of the event box are determined by shower distribution and inception frequency. coincide in space and time: a thunderstorm electric field, a hydrometeor that is large and elongated enough, and an extensive air shower creating a sufficient density of thermal electrons.

First, at least one hydrometeor of sufficient size and shape has to be inside the core of the air shower. For a core radius of $100 \mathrm{~cm}$ and a hydrometeor density of $10^{-7} \mathrm{~cm}^{-3}$, a height of $h \approx 3 \mathrm{~m}$ is sufficient. This limits the spatial height where the electric field has to exceed $2.7 \mathrm{kV} \mathrm{cm}^{-1}$ to $3 \mathrm{~m}$ as well.

Second, the air shower has to hit the horizontal area in the cloud where the electric field exceeds $2.7 \mathrm{kV} \mathrm{cm}^{-1}$. With at least five sufficiently energetic showers per $\mathrm{km}^{2}$ and per minute, we find at least one shower per second, if the horizontal high field area $\mathrm{A}$ is $12 \mathrm{~km}^{2}$, or equivalently 1 shower per minute, if the area is $0.2 \mathrm{~km}^{2}$.

Summary and discussion.-We have found that elongated hydrometeors of centimeter size are required to start a discharge at $5.5 \mathrm{~km}$ altitude, if the background field is as low as 0.15 times the classical breakdown field, and our simulations with a realistic permittivity $\epsilon(\omega)$ of ice showed that a streamer discharge actually can emerge, if 100 free electrons per $\mathrm{cm}^{3}$ are present. But due to electron attachment to oxygen and the formation of water clusters around these ions, free electrons are typically too rare for a discharge to start. However, a cosmic proton with energy above $5 \times 10^{15} \mathrm{eV}$ can create an extensive air shower whose core can provide the necessary electron density. These air showers appear with a frequency of 1 per $0.2 \mathrm{~km}^{2}$ per minute, and hence can explain how lightning discharges can start in an undervolted region. According to this argument, lightning inception at higher altitudes-say $12 \mathrm{~km}$-is less likely as the hydrometeor has to be larger and as the electron density in the extensive air shower is lower at these altitudes.

The strongest constraint in our analysis comes from the sizes of the hydrometeors which are little investigated within thunderclouds. Mason [26] suggested already in 1953 a correlation between graupel size and lightning inception - which should be studied further.

The height of the zone where the electric field has to exceed the runaway threshold is only of the size of meters, rather than of more than a kilometer as required for a relativistic runaway avalanche, and relativistic avalanching in an external electric field is not required in our model. Rather all free electrons are generated by the energy of the primary cosmic particle. The required height of the electric field zone is inversely proportional to the density distribution of large hydrometeors.

Finally, in our rare event analysis for the electron density in the shower core, we have focussed on a core radius of $1 \mathrm{~m}$. Future analysis might reveal even more favorable core radii and related primary particle energies.

A. D. acknowledges support by STW Project No. 12119 and C. R. and G. T. by FOM Project No. 12PR3041. 
*anna.dubinova@cwi.nl

†casper.rutjes@cwi.nl

[1] J. R. Dwyer and M. A. Uman, Electric field and charge structure in lightning-producing clouds, Phys. Rep. 534, 147 (2014).

[2] M. Stolzenburg and T. C. Marshall, Electric field and charge structure in lightning-producing clouds, in Lightning: Principles, Instruments and Applications: Review of Modern Lightning Research, edited by H. D. Betz, U. Schumann, and P. Laroche (Springer Science \& Business Media, New York, 2009), pp. 57-82.

[3] L. Coleman and J. Dwyer, Propagation speed of runaway electron avalanches, Geophys. Res. Lett. 33, L11810 (2006).

[4] J. Crabb and J. Latham, Corona from colliding drops as a possible mechanism for the triggering of lightning, Q. J. R. Meteorol. Soc. 100, 191 (1974).

[5] D. Petersen, M. Bailey, J. Hallett, and W. H. Beasley, Laboratory investigation of positive streamer discharges from simulated ice hydrometeors, Q. J. R. Meteorol. Soc. 132, 263 (2006).

[6] D. A. Petersen, M. Bailey, J. Hallett, and W. Beasley, Laboratory investigation of corona initiation by ice crystals and its importance to lightning, Q. J. R. Meteorol. Soc. 141, 1283 (2015).

[7] N. Liu, B. Kosar, S. Sadighi, J. R. Dwyer, and H. K. Rassoul, Formation of Streamer Discharges from an Isolated Ionization Column at Subbreakdown Conditions, Phys. Rev. Lett. 109, 025002 (2012).

[8] A. Sun, J. Teunissen, and U. Ebert, Why isolated streamer discharges hardly exist above the breakdown field in atmospheric air, Geophys. Res. Lett. 40, 2417 (2013).

[9] J. Teunissen, A. Sun, and U. Ebert, A time scale for electrical screening in pulsed gas discharges, J. Phys. D 47, 365203 (2014).

[10] M. Huertas, J. Fontan, and J. Gonzalez, Evolution times of tropospheric negative ions, Atmospheric Environment 12, 2351 (1978).

[11] I. Gallimberti, The mechanism of the long spark formation, J. Phys. (Paris), Colloq. 40, C7-193 (1979).

[12] A. V. Gurevich and A. N. Karashtin, Runaway Breakdown and Hydrometeors in Lightning Initiation, Phys. Rev. Lett. 110, 185005 (2013).
[13] K. Olive et al., Review of Particle Physics, Chin. Phys. C 38, 090001 (2014).

[14] E. Williams, Charge structure and geographical variation of thunderclouds in "The Lightning Flash," edited by V. Cooray (IEE, London, 2008), pp. 1-15.

[15] G. Hagelaar and L. Pitchford, Solving the Boltzmann equation to obtain electron transport coefficients and rate coefficients for fluid models, Plasma Sources Sci. Technol. 14, 722 (2005).

[16] Phelps database, www.lxcat.net, retrieved on August 31, 2014.

[17] L. D. Landau, J. Bell, M. Kearsley, L. Pitaevskii, E. Lifshitz, and J. Sykes, Electrodynamics of Continuous Media (Elsevier, New York, 1984), Vol. 8, pp. 39-42.

[18] D. Lamb and J. Verlinde, Physics and Chemistry of Clouds (Cambridge University Press, Cambridge, England, 2011), p. 26.

[19] G. Wormeester, S. Pancheshnyi, A. Luque, S. Nijdam, and U. Ebert, Probing photo-ionization: Simulations of positive streamers in varying $\mathrm{N}_{2}: \mathrm{O}_{2}$-mixtures, J. Phys. D 43, 505201 (2010).

[20] A. Luque, U. Ebert, C. Montijn, and W. Hundsdorfer, Photoionization in negative streamers: Fast computations and two propagation modes, Appl. Phys. Lett. 90, 081501 (2007).

[21] V. Artemov and A. Volkov, Water and Ice Dielectric Spectra Scaling at $0^{\circ} \mathrm{C}$, Ferroelectrics 466, 158 (2014).

[22] J. R. Dwyer and L. P. Babich, Low-energy electron production by relativistic runaway electron avalanches in air, J. Geophys. Res. 116, A09301 (2011).

[23] D. Heck, J. Knapp et al., CORSIKA: A Monte Carlo Code to Simulate Extensive Air Showers, (FZKA: Forschungszentrum Karlsruhe GmbH, Karlsruhe, 1998), Vol. 6019; www.ikp.kit .edu/corsika/.

[24] C. Köhn and U. Ebert, The structure of ionization showers in air generated by electrons with $1 \mathrm{MeV}$ energy or less, Plasma Sources Sci. Technol. 23, 045001 (2014).

[25] M. J. Berger, J. S. Coursey and J. Chang, NIST Database, Stopping Power Electrons, retrieved on Jan 2015 from www .nist.gov/pml/data/star/.

[26] B. Mason, On the generation of charge associated with graupel formation in thunderstorms, Q. J. R. Meteorol. Soc. 79, 501 (1953). 